\title{
Bioanalytical techniques in lipidomics
}

\author{
Dajana Vuckovic*,1,2,3 \\ ${ }^{1}$ Department of Chemistry \& Biochemistry, Concordia University, Montreal, Canada \\ ${ }^{2}$ Centre for Biological Applications of Mass Spectrometry, Concordia University, Montreal, Canada \\ ${ }^{3}$ PERFORM Centre, Concordia University, Montreal, Canada \\ * Author for correspondence: dajana.vuckovic@concordia.ca
}

First draft submitted: 16 January 2018; Accepted for publication: 19 January 2018; Published online: 13 March 2018

Keywords: lipidomics • lipids • mass spectrometry

Lipidomics can be defined as the comprehensive quantitative and qualitative analysis of the entire set of lipids present in a given organism. With the recent improvements in analytical technologies and improved coverage of lipidome, there is a resurgence of interest in understanding in detail biological roles of lipids, their regulation and dysregulation which may contribute to many diseases. The articles in this short themed issue of Bioanalysis highlight some of the analytical challenges and recent emerging technologies in lipidomics, with the focus on how to improve the analytical workflows for lipidomics. Currently, main analytical challenges in the field of lipidomics include:

- Difficulty in preserving lipidome at the time of sampling and completely stopping enzymatic and oxidation processes;

- Vast chemical diversity of lipids which precludes the use of a single extraction and analytical method to cover the entire lipidome;

- Low concentrations of many of lipids of interest, especially of bioactive lipids such as lipid mediators;

- Difficult structural elucidation and confirmation due to many possible isomeric species and lack of authentic standards for confirmation purposes.

This is by no means an exhaustive list but the articles in this themed issue discuss further many of these key challenges and set the basis to further advance this important field of inquiry.

In a Special Report, Vorkas discusses data-independent $\mathrm{MS}^{\mathrm{E}}$ acquisition strategy to facilitate lipid identification, with examples that include lipid class and fatty acid composition annotation [1]. The described protocol has been previously successfully applied to a wide variety of biological matrices including plasma, tissues and cells and can reliably detect more than 300 lipids in combination with methyl tert-butyl ether/methanol extraction. This special report summarizes briefly the capability and the limitations of the method and serves as an excellent introduction to the topic for the bioanalysts new to the lipidomics field. Vorkas argues that RP-UPLC-TOF-MS ${ }^{\mathrm{E}}$ is the tool of choice for lipidomics, a controversial claim certain to spark vigorous discussion in lipidomics community. The authors also show clear limitations of their workflow. For example, the protocol can be used to distinguish between some isomers within lysophospholipid classes. However, due to high fragmentation mass spectrum similarity and insufficient chromatographic separation, it is not possible to confidently assign structure of many other lipid isomers. Thus, both structural isomers and stereoisomers remain an extreme analytical challenge with additional approaches needed.

Research paper by Baker et al. describes how ion mobility spectrometry (IMS) can help resolve structural isomers of lipid mediators [2]. In this arena of bioactive signalling molecules, complete identification and distinguishing of isomers is critical, as different isomers can have entirely different biological roles. To illustrate the complexity of this analytical challenge, the authors cite a highly revealing example: more than 200 isomeric species from eicosanoid and isoprostane classes have formulas of $\mathrm{C}_{20} \mathrm{H}_{32} \mathrm{O}_{5}$ or $\mathrm{C}_{20} \mathrm{H}_{34} \mathrm{O}_{5}$ making their unequivocal identification and quantification extremely challenging in complex matrices. Chromatographic methods can help separate some of 
these species, usually at the expense of long analysis times and sample throughput, but additional high-throughput and orthogonal technologies are required to further supplement chromatographic separation. In this contribution, the authors examined the potential of IMS to separate 44 lipid mediator standards, and applied their optimized method to lung samples from animals exposed to different influenza strains. The authors found that positive ESI mode, where sodiated adducts are typically formed for many of lipid mediator classes, provide better isomer separation and clustering in IMS for some lipid subclasses. Collisional cross-sections acquired in this work provide important first step in further deployment and refinement of IMS strategies for lipid mediator analysis. The authors show that in positive ESI mode even some stereoisomers such as 12(S)-HETE and 12(R)-HETE can be separated with power of IMS. Of course, the need for positive ESI mode analysis to enhance IMS separations of some isomers raises new questions and analytical challenges: are endogenous levels of these lipid mediators sufficient to be detected in both modes? Using the infected lung samples, the authors indeed show that they can separate five of the most abundant (and overexpressed) HETE isomers from complex samples, as an exciting proof-of-concept study.

In the final Research Paper contribution for this thematic issue, Bowden et al. examine the effect of heattreatment on stabilization of lipids in tissues in three invertebrate species: earthworm, house cricket and ghost shrimp [3]. They provide convincing evidence that heat stabilization reduces phospholipase A and D activity, thus improving accuracy of lipidome analysis and perhaps make us re-examine the widespread use of the well-established flash-freezing using liquid $\mathrm{N}_{2}$ protocols.

Collectively, the works included in this thematic issue help to illustrate some of the key analytical challenges in lipidomics and propose the solution forward, while also inspiring new ideas. The final contribution to this themed issue also explores other uses of lipids, beyond the classical measurement of endogenous and exogenous lipids in biological matrices. More specifically, lipid nanoparticles can be used to enhance delivery of new drug therapies such as RNA-based drugs by addressing two challenges: increasing half-life of RNA in circulation by reducing accessibility to nuclease degradation and helping to cross cell membranes. These self-assembled lipid nanoparticles typically contain cationic lipid(s) which can interact with RNA, and combination of stabilizing lipids such as phosphatidylcholines and/or cholesterol. An Editorial by Hawthorne, Habach and Wilson discusses the measurement of cationic lipid as a surrogate for the measurement of lipid nanoparticle [4]. The main analytical challenge for this type of analysis is selective measurement of cationic lipid of interest in the presence of high endogenous background of phospholipids. This requires chromatographic separation and/or sample preparation, both of which are discussed in this editorial. The editorial also discusses best sample handling practices for collection of both biofluids and tissues for this determination. Finally, the main disadvantage of this approach is also highlighted: inability to distinguish between intact lipid nanoparticle and released cationic lipid. This opens up new research directions as to how to best map distribution of intact lipid nanoparticles, perhaps using technologies such as imaging MS.

I hope these insightful articles spark interest and discussion in lipidomics within bioanalytical community and look forward to hearing your feedback and comments on this themed issue. As a community, let us all make a New Year's resolution to critically examine our gold standard and favorite laboratory protocols and refine them when needed in order to improve the accuracy of our lipidome analyses and lipid identifications.

\section{Financial \& competing interests disclosure}

The authors have no relevant affiliations or financial involvement with any organization or entity with a financial interest in or financial conflict with the subject matter or materials discussed in the manuscript. This includes employment, consultancies, honoraria, stock ownership or options, expert testimony, grants or patents received or pending, or royalties.

No writing assistance was utilized in the production of this manuscript.

\section{References}

1 Vorkas PA. Expanding lipidome coverage using MS/MS-aided untargeted data-independent RP-UPLC-TOF-MSE acquisition. Bioanalysis 10(5), 307-319 (2018).

2 Kyle JE, Aly N, Zheng X, Burnum-Johnson KE, Smith RD, Baker ES. Evaluating lipid mediator structural complexity using Ion mobility spectrometry combined with mass spectrometry. Bioanalysis 10(5), 279-289 (2018).

3 Koelmel JP, Jones CM, Ulmer CZ et al. Examining heat treatment for stabilization of the lipidome. Bioanalysis 10(5), 291-305 (2018).

4 Hawthorne G, Harbach S, Wilson A. Cationic lipid bioanalysis: understanding distribution of lipid nanoparticles for delivery of RNA therapeutics. Bioanalysis 10(5), 275-277 (2018). 